\title{
Actitud del Profesorado Universitario Hacia la InClusión Educativa: UNA ReVISIÓN Sistemática ${ }^{1}$ \\ AtTitude of University Teachers ToWARds EDUCATIONAL INCLUSION: A SYSTEMATIC REVIEW
}

\author{
Eddy PAZ-MALDONADO ${ }^{2}$ \\ Hazaria FLORES-GIRÓN ${ }^{3}$
}

\begin{abstract}
RESUMEN: La actitud docente es determinante cuando se pretenden desarrollar espacios educativos inclusivos y atender a la diversidad. El objetivo de la presente revisión sistemática es analizar los estudios efectuados sobre la actitud del profesorado universitario hacia la inclusión educativa. Se incluyeron 15 artículos los cuales evidencian que en España se han realizado una mayor cantidad de investigaciones acerca del tema. Además, la perspectiva de inclusión planteada considera principalmente al alumnado en situación de discapacidad. También, se revela que el profesorado no está preparado para generar una enseńanza inclusiva. Referente a las actitudes, las investigaciones muestran hallazgos negativos y una resistencia por la implementación de adaptaciones curriculares. Los principales desafíos se encuentran relacionados con la preparación docente y la motivación por la constante capacitación. En conclusión, es necesario que los programas de formación de profesores consideren la inclusión educativa como una dimensión esencial que posibilite a los docentes exteriorizar actitudes positivas durante el quehacer pedagógico en las instituciones de Educación Superior.
\end{abstract}

PALABRAS CLAVE: Actitud. Conducta del profesor. Enseñanza Superior. Igualdad de oportunidades.

\begin{abstract}
The teaching attitude is decisive when trying to develop inclusive educational spaces and attend to diversity. The objective of this systematic review was to analyze the studies carried out on the attitude of university teachers towards educational inclusion. Fifteen articles were included, which show that in Spain a greater amount of research has been carried out on the subject. In addition, the perspective of the proposed inclusion mainly considers students in situation of disabilities. Also, it is revealed that teachers are not prepared to generate inclusive teaching. Regarding attitudes, research shows negative findings and resistance to the implementation of curricular adaptations. The main challenges are related to teacher preparation and motivation for constant training. In conclusion, it is necessary that teacher training programs consider educational inclusion as an essential dimension that enables teachers to express positive attitudes during pedagogical work in Higher Education institutions.
\end{abstract}

KEYWORDS: Attitude. Teacher behavior. Higher Education. Equal opportunity.

\section{INTRODUCCIÓN}

A lo largo del tiempo los centros de enseñanza superior han evolucionado, pues se ha permitido el ingreso del alumnado que forma parte de los distintos colectivos en condición de vulnerabilidad (Seale, 2017). Por ende, la inclusión educativa representa uno de los mayores desafíos para las universidades hoy en día. Desde esta perspectiva deben considerarse los actuales fenómenos relacionados con la migración, interculturalidad, atención al estudiantado con necesidades educativas especiales y otros aspectos a nivel social que tomen en cuenta la diversidad (Castillo Armijo \& Miranda Carvajal, 2018).

La inclusión educativa tiene el propósito de disminuir las brechas de desigualdad y opresión. Con su implementación se intenta instaurar un sistema educativo para todos con

\footnotetext{
${ }^{1}$ https://doi.org/10.1590/1980-54702021v27e0008

${ }^{2}$ Facultad de Humanidades y Artes. Departamento de Pedagogía y Ciencias de la Educación. Universidad Nacional Autónoma de Honduras. Tegucigalpa/Honduras. E-mail: eddy.paz@unah.edu.hn. ORCID: https://orcid.org/0000-0002-2324-8813

${ }^{3}$ Facultad de Odontología. Departamento de Estomatología. Universidad Nacional Autónoma de Honduras. Tegucigalpa/Honduras. E-mail: hazaria.flores@unah.edu.hn. ORCID: https://orcid.org/0000-0001-5131-9087
} 
base en la igualdad, participación y la no discriminación, con el fin de crear una sociedad verdaderamente democrática (Arnaiz Sánchez, 2003; Ibañez Martín et al., 2018). Este concepto nos traslada a la visión clásica de equidad educativa. Sin embargo, ha tomado mayor fuerza a nivel discursivo político-pedagógico debido al entorno de exclusión que prevalece actualmente (Calvo, 2013). Asimismo, puede considerarse como una pedagogía que reacciona ante la necesidad de enfrentar los dilemas de la diferencia (Del Pozo-Armentia et al., 2020).

En los entornos inclusivos la diversidad toma gran importancia (Moriña, 2017), puesto que se busca potenciar las capacidades de aprendizaje del alumnado, despertar su interés y ofrecer apoyos para que el proceso educativo sea efectivo (González-Rojas \& Triana-Fierro, 2018). La diversidad está ligada con características físicas, genéticas, culturales y personales que diferencian a los seres humanos y a los distintos colectivos sociales (Arnaiz Sánchez, 2003).

Con referencia al concepto actitud es uno de los más antiguos y controvertidos en las ciencias sociales. Las investigaciones realizadas acerca de este constructo se han centrado principalmente en su relación con los comportamientos (Laca, 2005). Escalante et al. (2012) la definen como disposición interna del ser humano hacia un individuo, objeto o situación. Bohner y Dickel (2011) establecen que es la evaluación de un objeto de pensamiento. Estos objetos están asociados con personas, grupos e ideas. Según Pacheco Ruíz (2002) es un elemento social, adquirido en los procesos de socialización y a la vez puede ser modificado representando un cambio social. Por su parte, Granada et al. (2013) mencionan que las actitudes son un conjunto de percepciones, creencias y sentimientos a favor o en contra de alguna circunstancia en particular. Además, son maneras de reaccionar ante la postura educativa que favorezca el logro de los aprendizajes de todo el estudiantado.

En la búsqueda de la inclusión educativa, las actitudes del profesorado representan un papel vital, se debe trabajar su desarrollo en el proceso de formación docente con la finalidad de aumentar las prácticas inclusivas y la calidad educacional. Las actitudes hacia la inclusión educativa son difíciles de abordar por la complejidad de los distintos factores que intervienen en ellas (Sanhueza et al., 2012), pero es necesario considerarlas en el contexto de la enseńanza inclusiva (Pegalajar Palomino \& Colmenero-Ruiz, 2014). Un profesorado universitario que muestra una actitud positiva es capaz de entender, respetar y aceptar la diversidad en los espacios formativos (Paz-Maldonado, 2018a). Estas actitudes positivas son esenciales para lograr el éxito educativo de todo el alumnado (Saloviita, 2020).

Las actitudes han sido objeto de estudio en distintos ámbitos, pero en lo que respecta al profesorado universitario y la inclusión educativa es un tema de reciente interés. Por consiguiente, el objetivo de la presente revisión sistemática es analizar los estudios efectuados sobre la actitud del profesorado universitario hacia la inclusión educativa. Consideramos que este trabajo es de gran importancia para avanzar en la búsqueda de una educación superior más inclusiva, debido a que permite profundizar en diversos aspectos planteados en las distintas investigaciones realizadas en el contexto global.

\section{Método}

El presente artículo de revisión sistemática siguió la declaración PRISMA (Urrútia \& Bonfill, 2010). Las revisiones sistemáticas posibilitan sintetizar información disponible de 
una temática, donde se pueden considerar elementos cuantitativos y cualitativos de los estudios primarios seleccionados en tal proceso (Manterola et al., 2013). Se formuló como pregunta de investigación: ¿Qué estudios han abordado la actitud del profesorado universitario hacia la inclusión educativa?

Se llevó a cabo una búsqueda principal el 13 de enero del año 2021 en las bases de datos Scopus, SciELO y Web of Science. Se utilizaron los descriptores: "actitud"; "profesores"; "educación superior" e "inclusión". En la Tabla 1 se detallan las estrategias de búsqueda utilizadas en cada una de las bases de datos. Además, se realizó una búsqueda complementaria manual en la base de datos Google Scholar.

\section{Tabla 1}

Estrategias de búsqueda en las bases de datos

\begin{tabular}{l|l}
\hline Scopus & $\begin{array}{l}\text { TITLE-ABS-KEY ((attitude) AND (teachers) AND ("higher education”) AND ( inclu- } \\
\text { sion }))\end{array}$ \\
\hline SciELO & (actitud) AND (profesores) AND (educación superior) AND (inclusión) \\
\hline Web of Science & TS=(attitude AND teachers AND “higher education” AND inclusion) \\
\hline
\end{tabular}

Los estudios incluidos en la revisión sistemática fueron aquellos a) artículos empíricos realizados en la educación superior que abordarán la actitud del profesorado universitario hacia la inclusión educativa, b) que entre sus participantes se considerarán a profesores universitarios y/o jefes administrativos, c) en los idiomas español, portugués e inglés y d) publicados del 1 ro de enero de 2012 al 31 de diciembre de 2020.

Se excluyeron a) artículos teóricos, revisiones, libros, reseñas, actas de congresos, tesis, b) estudios que sus participantes fueran únicamente estudiantes universitarios e c) investigaciones que abordarán la actitud del profesorado hacia la inclusión educativa en los niveles de educación Pre-básica, Básica y Media.

Los artículos identificados en la búsqueda principal y complementaria fueron revisados por los autores de este estudio, los cuales eliminaron aquellos artículos que estuvieran duplicados en las diversas bases de datos consultadas. Los artículos restantes se ordenaron en una tabla por año, autor, título y revista para ser evaluados por título, resumen y a texto completo.

Ambos autores evaluaron de forma independiente los artículos, donde aplicaron los criterios de inclusión y exclusión establecidos. Si el título o resumen de los artículos identificados mostraba uno o más criterios de exclusión, no se realizó ninguna evaluación adicional y el artículo fue descartado. Los artículos restantes fueron analizados a texto completo.

En la evaluación de los artículos ambos autores realizaron un consenso para determinar la elección. Cuando, existió desacuerdo entre ambos, referente a los artículos elegidos, se deliberó para alcanzar un acuerdo en la obtención de los artículos incluidos en la presente revisión sistemática.

Luego de seleccionar los artículos incluidos, se establecieron tres ejes temáticos: características de los estudios incluidos, la perspectiva de inclusión planteada en las investigaciones analizadas y la actitud del profesorado hacia la inclusión educativa. El propósito fue realizar 
un análisis exhaustivo de las diferentes publicaciones considerando principalmente los aspectos sobre la actitud del profesorado universitario hacia la inclusión educativa.

En la Figura 1 se muestra el diagrama de flujo PRISMA sobre la selección de artículos incluidos en esta revisión sistemática.

\section{Figura 1}

Diagrama de flujo de la selección de artículos de acuerdo con PRISMA.
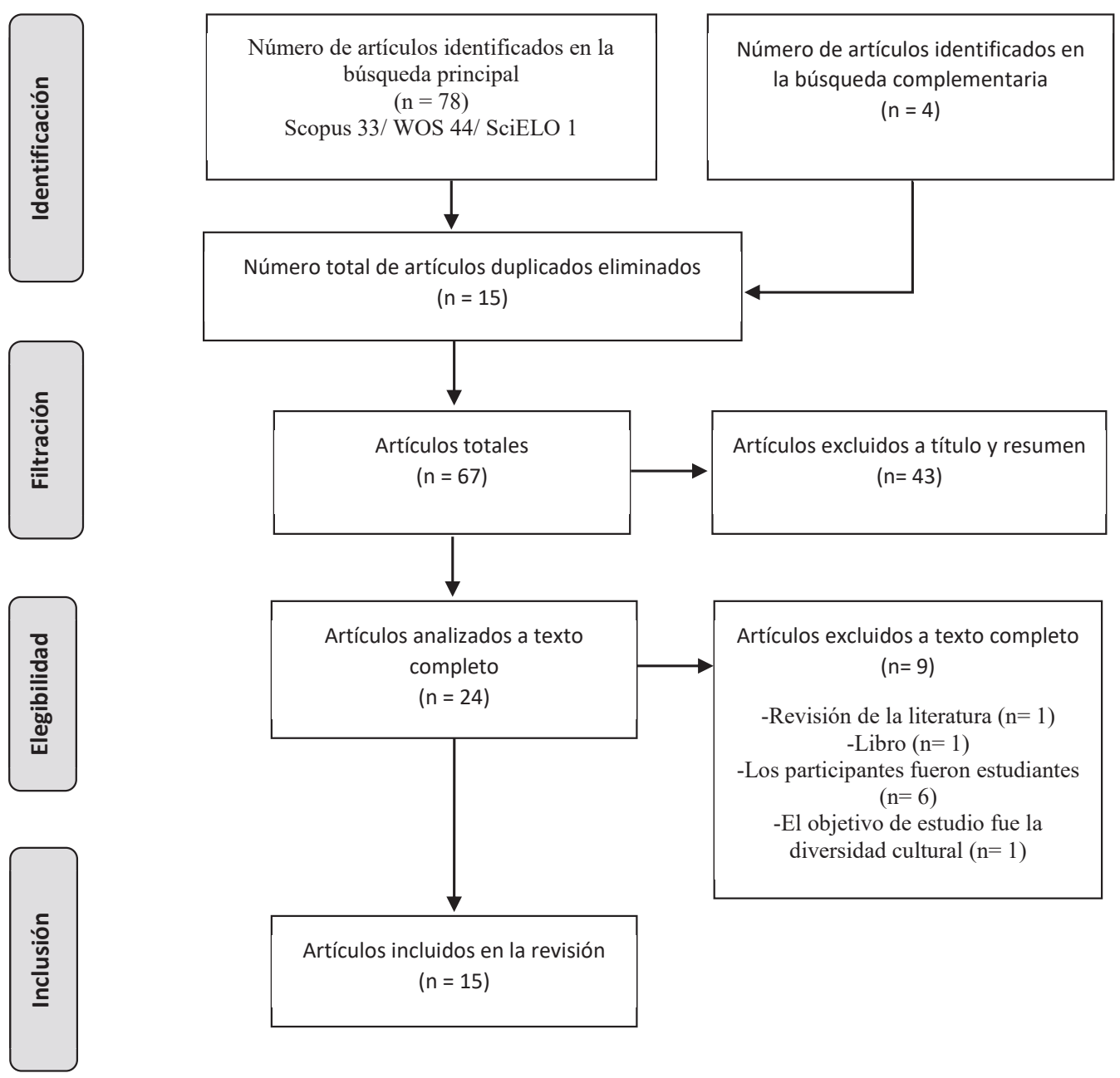

\section{Resultados}

Con el propósito de dar respuesta al objetivo y a la pregunta de investigación establecida en esta revisión sistemática, se presentan los resultados agrupados en tres ejes temáticos. El artículo analiza las distintas características de los estudios incluidos, la perspectiva de inclusión planteada y la actitud del profesorado hacia la inclusión educativa. 


\subsection{CaRACTERÍsticas de los ESTUdios INCLUIDOS}

Se incluyeron 15 artículos publicados entre los años 2012 al 2020. La mayoría de los estudios incluidos se realizaron en Espańa (7) y Chile (2). Por otra parte, el abordaje metodológico empleado en 12 de los estudios fue de tipo cuantitativo y en 3 de ellos se utilizó un enfoque cualitativo. En la Tabla 2 se describen las características y en la Tabla 3 se brindan los datos descriptivos de los estudios incluidos en esta revisión sistemática.

\section{Tabla 2}

\section{Características de los estudios incluidos}

\begin{tabular}{l|c|l|l}
\hline \multicolumn{1}{c|}{ Autores } & \multicolumn{1}{c|}{$\begin{array}{c}\text { Ańo de } \\
\text { publicación }\end{array}$} & \multicolumn{1}{c}{$\begin{array}{c}\text { Lugar de realización } \\
\text { del estudio }\end{array}$} & \multicolumn{1}{|c}{ Indexación } \\
\hline Ahsan et al. & 2012 & Bangladesh & Scopus \\
\hline Álvarez-Pérez y López-Aguilar & 2015 & España & Google Scholar \\
\hline Bell-Rodríguez & 2020 & Ecuador & Scopus \\
\hline Davy et al. & 2015 & Reino Unido & Web of Science \\
\hline Emmers et al. & 2020 & Bélgica & Scopus \\
\hline Garabal-Barbeira & 2015 & España & Google Scholar \\
\hline Garabal-Barbeira et al. & 2018 & España & Web of Science \\
\hline Herrera-Seda et al. & 2016 & Chile & Google Scholar \\
\hline Ke et al. & 2017 & Rusia & Web of Science \\
\hline Llorent et al. & 2020 & España & Web of Science \\
\hline Ocampo & 2012 & Chile & Google Scholar \\
\hline Ortiz Colón et al. & 2018 & España & Web of Science \\
\hline Poker et al. & 2018 & Brasil & SciELO \\
\hline Polo Sánchez et al. & 2017 & España & Scopus \\
\hline Rodríguez-Martín et al. & 2014 & España & Scopus \\
\hline
\end{tabular}

Tabla 3

Datos descriptivos de los estudios incluidos

\begin{tabular}{l|l|l|l}
\hline \multicolumn{1}{c|}{ Autores/año } & \multicolumn{1}{c|}{ Objetivo del estudio } & \multicolumn{1}{c}{$\begin{array}{c}\text { Tipo de } \\
\text { estudio }\end{array}$} & \multicolumn{1}{c}{ Participantes } \\
\hline \multirow{2}{*}{$\begin{array}{l}\text { Ahsan et al., 2012 } \\
\text {-Aguilar, 2015 }\end{array}$} & $\begin{array}{l}\text { Revelar las creencias de los jefes de institucio- } \\
\text { nes de educación superior sobre los desafíos } \\
\text { que enfrentan al preparar a los docentes en } \\
\text { formación para generar la inclusión educativa } \\
\text { en Bangladesh. }\end{array}$ & Cualitativo & 22 jefes administrativos \\
\hline $\begin{array}{l}\text { Analizar el rol que desempeña el profesora- } \\
\text { do en la puesta en práctica de un enfoque } \\
\text { inclusivo de la enseñanza y las dificultades que } \\
\text { encuentra en su labor educativa y orientadora. }\end{array}$ & Cuantitativo & $\begin{array}{l}111 \text { profesores universi- } \\
\text { tarios }\end{array}$ \\
\hline
\end{tabular}




\begin{tabular}{|c|c|c|c|}
\hline Bell-Rodríguez, 2020 & $\begin{array}{l}\text { Describir las distintas fases que conforman el } \\
\text { ciclo para la inclusión educativa en la educaci- } \\
\text { ón superior, como premisa para la promoción } \\
\text { de la reflexión y de las transformaciones que } \\
\text { respondan a las exigencias para su estableci- } \\
\text { miento y adecuado funcionamiento. }\end{array}$ & Cualitativo & $\begin{array}{l}8 \text { estudiantes en situación } \\
\text { de discapacidad, } 12 \text { pro- } \\
\text { fesores universitarios y } 11 \\
\text { familiares de tal alumnado }\end{array}$ \\
\hline Davy et al., 2015 & $\begin{array}{l}\text { Analizar las barreras y los facilitadores para } \\
\text { proporcionar contenido curricular de atención } \\
\text { médica, de salud y social LGBT que figuran en } \\
\text { las políticas de acreditación y dentro de la en- } \\
\text { seńanza de medicina en pregrado y posgrado. }\end{array}$ & Cualitativo & 10 profesores universitarios \\
\hline Emmers et al., 2020 & $\begin{array}{l}\text { Explorar la relación entre actitudes, autoefi- } \\
\text { cacia y el comportamiento del profesorado } \\
\text { de Educación Superior hacia el alumnado en } \\
\text { situación de discapacidad. }\end{array}$ & Cuantitativo & 75 profesores universitarios \\
\hline Garabal-Barbeira, 2015 & $\begin{array}{l}\text { Determinar las actitudes de alumnos y } \\
\text { docentes hacia los estudiantes en situación de } \\
\text { discapacidad. }\end{array}$ & Cuantitativo & $\begin{array}{l}121 \text { profesores y } 345 \text { estu- } \\
\text { diantes universitarios }\end{array}$ \\
\hline $\begin{array}{l}\text { Garabal-Barbeira et al., } \\
2018\end{array}$ & $\begin{array}{l}\text { Determinar las actitudes del alumnado y } \\
\text { docentes hacia la discapacidad y conocer las } \\
\text { variables que influyen en dichas actitudes. }\end{array}$ & Cuantitativo & $\begin{array}{l}121 \text { profesores y } 345 \text { estu- } \\
\text { diantes universitarios }\end{array}$ \\
\hline Herrera-Seda et al., 2016 & $\begin{array}{l}\text { Indagar las características psicométricas de dos } \\
\text { instrumentos que permiten recoger informa- } \\
\text { ción acerca de la promoción de la inclusión } \\
\text { educativa por parte de docentes universitarios. }\end{array}$ & Cuantitativo & $\begin{array}{l}232 \text { profesores universi- } \\
\text { tarios }\end{array}$ \\
\hline Ke et al., 2017 & $\begin{array}{l}\text { Evaluar la preparación psicológica de los } \\
\text { docentes para trabajar con estudiantes con } \\
\text { oportunidades de salud limitadas. }\end{array}$ & Cuantitativo & 19 profesores universitarios \\
\hline Llorent et al., 2020 & $\begin{array}{l}\text { Determinar la percepción del profesorado } \\
\text { sobre la educación inclusiva en la universidad. }\end{array}$ & Cuantitativo & $\begin{array}{l}1,252 \text { profesores univer- } \\
\text { sitarios }\end{array}$ \\
\hline Ocampo, 2012 & $\begin{array}{l}\text { Reflexionar en torno a los desafíos y las } \\
\text { oportunidades que enfrenta la inclusión de es- } \\
\text { tudiantes en situación de discapacidad respecto } \\
\text { de su acceso y participación de experiencias en } \\
\text { educación superior. }\end{array}$ & Cuantitativo & $\begin{array}{l}25 \text { profesores universita- } \\
\text { rios, } 65 \text { estudiantes y } 10 \\
\text { autoridades del gobierno } \\
\text { universitario }\end{array}$ \\
\hline Ortiz Colón et al., 2018 & $\begin{array}{l}\text { Analizar la percepción del personal docente } \\
\text { de la Universidad de Jaén con respecto a la } \\
\text { integración de los estudiantes en situación de } \\
\text { discapacidad, y describir las intervenciones } \\
\text { que utilizan para responder a las necesidades } \\
\text { específicas de estos estudiantes, para examinar } \\
\text { las diferencias que existen en intervenciones de } \\
\text { docentes para estudiantes en esta condición en } \\
\text { función de su facultad. }\end{array}$ & Cuantitativo & $\begin{array}{l}300 \text { profesores universi- } \\
\text { tarios }\end{array}$ \\
\hline Poker et al., 2018 & $\begin{array}{l}\text { Indagar y analizar las percepciones de los do- } \\
\text { centes de una universidad sobre el proceso de } \\
\text { inclusión de estudiantes con discapacidades. }\end{array}$ & Cuantitativo & 52 profesores universitarios \\
\hline
\end{tabular}




\begin{tabular}{l|l|l|l}
\hline & $\begin{array}{l}\text { Analizar la actitud hacia la inclusión de estu- } \\
\text { diantes con discapacidades en el área del aula } \\
\text { universitaria, enfatizando la importancia de las } \\
\text { actitudes de los docentes, pero también la del } \\
\text { Pesto de la comunidad universitaria en general } \\
\text { para que dicha inclusión sea exitosa. }\end{array}$ & Cuantitativo & $\begin{array}{l}2521 \text { estudiantes sin disca- } \\
\text { pacidad, 33 estudiantes en } \\
\text { situación de discapacidad, } \\
79 \text { profesores universitarios } \\
\text { y 38 miembros del personal } \\
\text { administrativo y de servicio }\end{array}$ \\
\hline \multirow{2}{*}{$\begin{array}{l}\text { Rodríguez-Martín et al., } 2017 \\
2014\end{array}$} & $\begin{array}{l}\text { Conocer las actitudes hacia la inclusión aca- } \\
\text { démica y social de los estudiantes en situación } \\
\text { de discapacidad que tiene el profesorado y } \\
\text { los estudiantes del área de educación de dos } \\
\text { facultades y universidades distintas. }\end{array}$ & Cuantitativo & $\begin{array}{l}85 \text { profesores y } 1015 \text { estu- } \\
\text { diantes universitarios }\end{array}$ \\
\hline
\end{tabular}

\subsection{PERSPECTIVA DE INCLUSIÓN PLANTEADA EN LAS INVESTIGACIONES INCLUIDAS}

En 10 de los estudios incluidos se aborda la inclusión dirigida únicamente hacia el estudiantado en situación de discapacidad. Estas investigaciones plantean aspectos vinculados con el establecimiento de programas de becas, adaptación de materiales, autoeficacia docente, participación del alumnado, formación del profesorado, desarrollo de prácticas pedagógicas positivas, creación de normativas universitarias, elaboración de planes de atención y la implementación de adaptaciones curriculares (Bell-Rodríguez, 2020; Emmers et al., 2020; GarabalBarbeira, 2015; Garabal-Barbeira et al., 2018; Ke et al., 2017; Ocampo, 2012; Ortiz Colón et al., 2018; Poker et al., 2018; Polo Sánchez et al., 2017; Rodríguez-Martín et al., 2014).

Por otra parte, 4 trabajos consideran una perspectiva más amplia de inclusión educativa. Esta visión centrada particularmente en el desarrollo de espacios educativos inclusivos donde se señalan elementos como: la preocupación por la idea de inclusión desarrollada, preparación docente para atender la diversidad, respuesta educativa a las necesidades del estudiantado y las prácticas educativas inclusivas (Ahsan et al., 2012; Álvarez-Pérez \& López-Aguilar, 2015; Herrera-Seda et al., 2016; Llorent et al., 2020).

Finalmente, solo 1 investigación considera la inclusión de la diversidad sexual, el estudio aborda específicamente las barreras y facilitadores existentes para proporcionar contenido curricular LGTB en programas de enseñanza de medicina a nivel de pregrado y posgrado (Davy et al., 2015).

\subsection{ACTITUdES DEL PROFESORADO HACIA LA INCLUSIÓN EDUCATIVA}

Referente a las actitudes que muestra el profesorado para generar la inclusión educativa en los espacios áulicos universitarios, 7 de los estudios exponen resultados negativos que impactan en el proceso de enseñanza (Álvarez-Pérez \& López-Aguilar, 2015; Davy et al., 2015; Garabal-Barbeira et al., 2018; Garabal-Barbeira, 2015; Ke et al., 2017; Ortiz Colón et al., 2018; Rodríguez-Martín et al., 2014). Estas actitudes negativas se exteriorizan en elementos como: la escasa preparación docente que posibilite brindar respuestas educativas mediante sus actuaciones dentro y fuera del aula (Álvarez-Pérez \& López-Aguilar, 2015; Ortiz Colón et al., 2018), falta de enfoque acerca de distintas temáticas, por ejemplo, la diversidad sexual (Davy et al., 2015), la no priorización de adaptaciones curriculares para atender al estudiantado que lo requiera (Garabal-Barbeira, 2015; Garabal-Barbeira et al., 2018; Rodríguez-Martín et al., 
2014). Otro elemento es la insuficiente competencia psicológica vinculada con la empatía y tolerancia en torno a los estudiantes en situación de discapacidad (Ke et al., 2017).

Por otra parte, 5 investigaciones dieron a conocer actitudes positivas demostradas por el profesorado universitario como ser: la utilización de diferentes materiales y tipos de evaluación en el quehacer pedagógico con el fin de dar respuesta a las necesidades de los educandos (Bell-Rodríguez, 2020), el optimismo por la constante mejora educativa (Herrera-Seda et al., 2016) que posibilita implicarse en el uso de diversas estrategias pedagógicas, el desarrollo de una visión de inclusión como elemento de igualdad en el plano personal y social, sin reproducir un paradigma ligado con el sufrimiento (Ocampo, 2012), un trato igualitario entre docente-estudiante favoreciendo el diálogo, el respeto y la equidad (Poker et al., 2018). También, una concepción positiva hacia el alumnado, erradicando toda perspectiva de segregación e inferioridad (Polo Sánchez et al., 2017).

Asimismo, 1 de los estudios proyecta tanto resultados negativos como positivos. En primer lugar, evidencia que los jefes administrativos de universidades que forman a profesionales de la educación demostraron una actitud negativa con respecto al tema de la inclusión educativa. Este aspecto tiene un impacto perjudicial en las creencias y percepciones de los futuros docentes. En un segundo momento, sostiene la importancia de valorar e impulsar la inclusión educativa en la formación inicial para garantizar la igualdad de derechos a todo el estudiantado (Ahsan et al., 2012).

Además, 2 de los estudios obtuvieron resultados neutrales referentes a las actitudes del profesorado hacia la inclusión educativa (Emmers et al., 2020; Llorent et al., 2020). Esto demuestra que mujeres y hombres participantes en dichas investigaciones tienen igual nivel de formación en materia de inclusión educativa (Llorent et al., 2020). Por otro lado, se plantea que existen una serie de factores como: la edad, el género y la experiencia profesional que condicionan la actitud demostrada por los docentes (Emmers et al., 2020).

En relación con la implementación de adaptaciones curriculares hacia el estudiantado en situación de discapacidad, 3 de los estudios reflejan actitudes negativas en la falta de utilización de adaptaciones curriculares en los espacios de enseñanza-aprendizaje. Existen docentes que consideran innecesario adecuar los contenidos (Garabal-Barbeira, 2015; Garabal-Barbeira et al., 2018), objetivos, actividades y criterios de evaluación. Mostrando una actitud negativa para realizar dichas adecuaciones (Rodríguez-Martín et al., 2014). En cambio, otros están de acuerdo con adaptar solamente materiales educativos utilizados en diferentes momentos (Garabal-Barbeira et al., 2018). En estas investigaciones, se puede visualizar que el profesorado universitario demuestra actitudes negativas y escasa voluntad para desarrollar acciones que permitan apoyar a los estudiantes en situación de discapacidad durante su proceso formativo (Garabal-Barbeira, 2015; Garabal-Barbeira et al., 2018; Rodríguez-Martín et al., 2014).

Por el contrario, 1 de los estudios expone que los estudiantes en situación de discapacidad, presentaron una baja solicitud dirigida hacia el profesorado para adaptar la metodología utilizada en los diferentes espacios de aprendizaje de las carreras universitarias a las que pertenecen. Además, en la investigación se menciona que los docentes tienen mayores dificultades para brindar atención al alumnado con discapacidades visuales e intelectuales, debido al desconocimiento de las mismas (Ortiz Colón et al., 2018). 


\subsubsection{Preparación del Profesorado para generar una ENSEŃanza inClusiva}

Los procesos formativos iniciales deben contribuir con la preparación del profesorado con el propósito de generar una enseńanza inclusiva (Ahsan et al., 2012). En ocasiones los docentes universitarios no han sido formados para ofrecer una respuesta educativa a la diversidad (Álvarez-Pérez \& López-Aguilar, 2015). La investigación realizada por Ahsan et al. (2012) plantea que las mayores preocupaciones del profesorado son su preparación y el cambio de actitudes hacia la inclusión educativa. Este cambio de actitud se vuelve más complejo cuando se tienen prejuicios sobre algunos grupos sociales (Davy et al., 2015).

En el caso del estudiantado en situación de discapacidad 5 estudios afirman que el profesorado no está preparado para trabajar con dicho alumnado (Álvarez-Pérez \& LópezAguilar, 2015; Bell-Rodríguez, 2020; Ocampo, 2012; Ortiz Colón et al., 2018; Poker et al., 2018), dado que demuestra inseguridad (Poker et al., 2018). Por tanto, se requiere la formación en este ámbito (Álvarez-Pérez \& López-Aguilar, 2015; Ocampo, 2012) con el fin de que previo al inicio del año académico se lleve a cabo una socialización de las discapacidades que presentan los estudiantes y que sean los docentes los que puedan plantear acciones con el propósito de generar una enseñanza inclusiva. Es fundamental tomar en cuenta que las actitudes hacia la inclusión educativa son importantes para lograr la permanencia académica de los educandos en esta condición (Bell-Rodríguez, 2020).

De igual forma, es vital desarrollar competencias psicológicas que faciliten brindar apoyo pedagógico a los estudiantes en situación de discapacidad (Ke et al., 2017). Sin embargo, el desconocimiento en la materia ocasiona una serie de acciones negativas que influyen en el proceso educativo (Rodríguez-Martín et al., 2014). Existen 2 trabajos que realizaron comparaciones entre profesoras y profesores de distintas facultades. En el primero, el profesorado de Ciencias de la Salud tuvo mayores dificultades al momento de contar con educandos en esta condición en los espacios de aprendizaje. En cambio, los docentes de Humanidades y Ciencias de la Educación presentaron menores problemas (Ortiz Colón et al., 2018). En cuanto al segundo estudio, se reveló que el profesorado de Ciencias de la Educación utilizó con mayor frecuencia una didáctica inclusiva para atender a todo el alumnado en comparación con el grupo de profesores de las Ciencias Experimentales (Llorent et al., 2020).

Por otro lado, 5 investigaciones ofrecen resultados positivos asociados con la preparación del profesorado para posibilitar la participación, igualdad de oportunidades, consideración, sensibilidad y autoeficacia en el proceso de enseñanza-aprendizaje (Emmers et al., 2020; Garabal-Barbeira et al., 2018; Garabal-Barbeira, 2015; Herrera-Seda et al., 2016; Polo Sánchez et al., 2017). No obstante, 1 de los estudios menciona que las profesoras tienen mayores puntajes en comparación con los profesores, debido a que utilizan una didáctica inclusiva y una mejor organización del centro educativo, al momento de desarrollar su quehacer docente (Llorent et al.,2020).

\subsubsection{Desafíos PARA GENERAR LA INCLUSIÓN EDUCATIVA}

Generar la inclusión educativa en la enseñanza superior implica afrontar una serie de desafíos relativos a la formación inicial del profesorado que labora en los centros educativos y la motivación de este hacia la constante capacitación en dicha materia (Ahsan et al., 2012), 2 de 
los estudios dan a conocer la falta de preparación en esta etapa formativa (Ahsan et al., 2012; Poker et al., 2018). También, 5 de las investigaciones seńalan que debe capacitarse a los docentes en el uso de estrategias y recursos que les permita ofrecer una atención personalizada a todo el alumnado (Álvarez-Pérez \& López-Aguilar, 2015; Garabal-Barbeira et al., 2018; GarabalBarbeira, 2015; Llorent et al.,2020; Ortiz Colón et al., 2018). De igual manera, 2 de los trabajos se refieren a que es necesario analizar con mayores detalles diversas temáticas entre ellas: la discapacidad y la diversidad sexual (Bell-Rodríguez, 2020; Davy et al., 2015). Comprender estos y otros temas contribuirá con la producción de actitudes más positivas (Emmers et al., 2020), concepciones y prácticas inclusivas (Herrera-Seda et al., 2016).

La universidad es un espacio donde debe impulsarse la creación de normativas, políticas inclusivas y planes de atención dirigidos a todo el alumnado que lo demande (Ocampo, 2012). En ese sentido, las instituciones de educación superior tienen la obligación de ofrecer apoyos al profesorado con la finalidad de que este pueda atender la diversidad en los diferentes espacios de enseńanza-aprendizaje (Ke et al., 2017; Poker et al., 2018). Uno de estos apoyos son los programas de formación docente y asesoramiento para la comunidad universitaria con el propósito de fomentar actitudes positivas hacia la inclusión educativa, tal como lo proponen dos de los estudios revisados (Polo Sánchez et al., 2017; Rodríguez-Martín et al., 2014).

\section{Discusiones y CONCLUSIÓN}

El objetivo de este artículo fue analizar mediante una revisión sistemática los estudios efectuados sobre la actitud del profesorado universitario hacia la inclusión educativa. De acuerdo con los resultados evidenciados en este trabajo, España es el país donde se han realizado un mayor número de investigaciones sobre la actitud del profesorado universitario hacia la inclusión educativa, seguido por Chile. Casi en su totalidad los estudios utilizaron una metodología cuantitativa y en la mayoría los participantes fueron profesores universitarios. Sin embargo, 1 de las investigaciones contó con la participación de jefes administrativos de carreras relacionadas con la formación docente (Ahsan et al., 2012).

La perspectiva sobre inclusión mostrada en gran parte de las investigaciones incluidas, abordan únicamente al alumnado en situación de discapacidad. Solo 4 estudios consideraron la inclusión educativa desde una visión amplia (Ahsan et al., 2012; Álvarez-Pérez \& LópezAguilar, 2015; Herrera-Seda et al., 2016; Llorent et al., 2020). Por consiguiente, es necesario comprender la inclusión como aquella que permita democratizar los espacios de aprendizaje, valorar las diferencias y brindar atención a todo el estudiantado que asiste regularmente a los centros de educación superior (Paz-Maldonado, 2020a). Esta concepción permitirá que los docentes puedan implementar metodologías educativas inclusivas entre ellas: el aprendizaje cooperativo, los grupos interactivos y el aprendizaje dialógico (Azorín Abellán, 2018; Lata \& Castro, 2016; Tejeda Cerda, 2019), para la eliminación de las barreras en la enseñanza universitaria (Sandoval Mena et al., 2019).

En lo relativo a las actitudes del profesorado hacia la inclusión educativa se registra un mayor número de estudios que declaran la existencia de actitudes negativas por parte de los docentes universitarios. Dichas actitudes se expresan desde la limitada preparación docente para prestar atención al alumnado hasta la falta de empatía y tolerancia. Estos resultados son 
similares a otras investigaciones donde se indica que los profesores tienen menores puntuaciones en el desarrollo de prácticas inclusivas durante su quehacer pedagógico (Capelli et al., 2020; González-Gil et al., 2016). Por otro lado, una menor cantidad de artículos mostraron actitudes positivas, lo que denota una mejor formación e interés del profesorado para promover una enseñanza inclusiva dirigida a todo el estudiantado. Los hallazgos encontrados concuerdan con distintos trabajos realizados concernientes a la temática (Avramidis \& Norwich, 2002; González-Castellano et al., 2021; Moriña, 2020; Sánchez Díaz \& Morgado Camacho, 2021).

Este panorama revela que el profesorado se convierte en un facilitador o una barrera en el proceso de enseńanza-aprendizaje. La actitud mostrada por los docentes hacia la inclusión educativa es un aspecto intrínseco, que requiere transformar paradigmas negativos en nuevas oportunidades. La actitud promueve la participación del estudiantado o la reduce significativamente (Angenscheidt Bidegain \& Navarrete Antola, 2017) y es determinante para llevar a cabo prácticas educativas inclusivas (Granada et al., 2013; Paz-Maldonado, 2020b), por lo que se vuelve transcendental ejecutar acciones para cambiar las actitudes del profesorado (Yada \& Savolainen, 2017). Las actitudes positivas de los docentes son básicas para implementar la inclusión educativa (De Boer et al., 2011), pues posibilitan el uso de estrategias inclusivas en el quehacer docente (Garzón et al., 2016). Las actitudes están condicionadas por el contexto en el que se desenvuelve el profesorado independientemente del área académica a la que pertenezca (Rodríguez-Martín \& Álvarez-Arregui, 2015).

Con respecto a la implementación de adaptaciones curriculares en su mayoría los estudios revisados dieron a conocer que hay resistencia y una actitud negativa por parte del profesorado. Similares hallazgos brindan otras investigaciones que ofrecen visiones controversiales (Hernández Garre et al., 2019; Navarro-Aburto et al., 2016; Rodríguez-Martín \& ÁlvarezArregui, 2015; Sevilla Santo et al., 2017).

Por lo que se refiere a la preparación del profesorado para generar una enseñanza inclusiva, es categórico que los docentes universitarios desarrollen una serie de competencias que les facilite atender la diversidad en los espacios de aprendizaje (Fernández Batanero, 2013; Paz-Maldonado, 2018a). Por tanto, los principios de la inclusión educativa deben incorporarse en los programas de formación docente inicial y permanente (Paz-Maldonado, 2018b). Es a través de la academia que se podrán abordar distintos temas que permitan al profesorado reflexionar para su mejora continua y alcanzar una enseñanza más inclusiva (De Haro Rodríguez et al., 2020).

Lograr implementar una enseñanza inclusiva en la educación superior demanda la preparación del profesorado en temas referentes al estudiantado en situación de discapacidad, pueblos indígenas, afrodescendientes, diversidad sexual y los diferentes colectivos en condición de vulnerabilidad que asisten regularmente a los centros educativos. Las universidades actualmente, necesitan docentes sensibilizados y con un amplio conocimiento de diversos mecanismos para impulsar la inclusión educativa. Por tanto, la formación del profesorado es uno de los grandes retos planteados (Álvarez Castillo \& Buenestado Fernández, 2015; Infante, 2010; Pegalajar Palomino \& Colmenero-Ruiz, 2014; Pereira et al., 2020). La universidad se considera un espacio de fomento de la inclusión educativa, promotora de igualdad de oportunidades y de actitudes positivas (González Cortés \& Roses Campos, 2016). Por ende, generar la inclusión 
educativa en los establecimientos de este nivel, implica poner atención en múltiples aspectos como ser: las actitudes del profesorado, las prácticas pedagógicas, la motivación hacia la enseñanza, la formación inicial y permanente como parte del ejercicio pedagógico.

Las limitaciones de este trabajo estuvieron relacionadas con la escasa producción científica acerca de la actitud del profesorado universitario hacia la inclusión educativa desde una perspectiva amplia, puesto que los estudios mayoritariamente referían al estudiantado en situación de discapacidad. Por consiguiente, el artículo busca ser un insumo para las futuras investigaciones que se pretendan realizar. Se sugiere continuar profundizando en las acciones que llevan a cabo los docentes con la finalidad de atender la diversidad, los programas de formación docente, las percepciones hacia la inclusión educativa, las prácticas pedagógicas, las adaptaciones curriculares y otros elementos esenciales referidos con el tema.

En conclusión, los estudios revisados permiten afirmar que la actitud docente es un elemento determinante en el proceso de enseńanza-aprendizaje. Es fundamental que los programas de formación del profesorado consideren como una dimensión esencial la preparación en materia de inclusión educativa desde una visión más amplia que incluya a todos los colectivos en condición de vulnerabilidad que participan en las instituciones de educación superior. De igual manera, las universidades deben implementar una enseñanza inclusiva que involucre y facilite la participación de toda la comunidad universitaria, brindar apoyos, generar condiciones para el desarrollo de este aspecto, valorar las diferencias y evitar los prejuicios que generan exclusión principalmente hacia aquellos grupos que han sido marginados históricamente.

\section{REFERENCIAS}

Ahsan, M. T., Sharma, U., \& Deppeler, J. M. (2012). Challenges to prepare pre-service teachers for inclusive education in Bangladesh: beliefs of higher educational institutional heads. Asia Pacific Journal of Education, 32(2), 241-257. https://doi.org/10.1080/02188791.2012.655372

Álvarez Castillo, J., \& Buenestado Fernández, M. (2015). Predictores de las actitudes hacia la inclusión de alumnado con necesidades educativas especiales en futuros profesionales de la educación. Revista Complutense de Educación, 26(3), 627-645. https://doi.org/10.5209/rev_RCED.2015.v26. $\mathrm{n} 3.44551$

Álvarez-Pérez, P. R., \& López-Aguilar, D. (2015). Atención del profesorado universitario a estudiantes con necesidades educativas específicas. Educación y Educadores, 18(2), 193-208. http://dx.doi. org/10.5294/edu.2015.18.2.1

Angenscheidt Bidegain, L., \& Navarrete Antola, I. (2017). Actitudes de los docentes acerca de la educación inclusiva. Ciencias Psicológicas, 11(2), 233-243. http://dx.doi.org/10.22235/cp.v11i2.1500

Arnaiz Sánchez, P. (2003). Multiculturalidad y diversidad en las aulas. Indivisa - Boletín de Estudios e Investigación, 4, 9-30.

Avramidis, E., \& Norwich, B. (2002). Teachers' attitudes towards integration / inclusion: a review of the literature. European Journal of Special Needs Education, 17(2), 129-147. https://doi. org/10.1080/08856250210129056

Azorín Abellán, C. (2018). El método de aprendizaje cooperativo y su aplicación en las aulas. Perfiles Educativos, 40(161), 181-194. 
Bell-Rodríguez, R. F. (2020). Comprensión del ciclo para la inclusión educativa en la educación superior ecuatoriana. Revista Electrónica Educare, 24(1), 1-21. https://doi.org/10.15359/ree.24-1.4

Bohner, G., \& Dickel, N. (2011). Attitudes and Attitude Change. Annual Review of Psychology, 62(1), 391-417. https://doi.org/10.1146/annurev.psych.121208.131609

Calvo, G. (2013). La formación de docentes para la inclusión educativa. Páginas de Educación, 6(1), $19-35$.

Capelli, J. C. S., Di Blasi, F., \& Dutra, F. B. S. (2020). Professors' perception of the entry of a deaf student into a university campus. Revista Brasileira de Educação Especial, 26(1), 67-88.

Castillo Armijo, P., \& Miranda Carvajal, C. (2018). Actitud hacia la Inclusión de los Estudiantes de Pedagogía de una Universidad Estatal Chilena. Revista Latinoamericana de Educación Inclusiva, 12(2), 133-148. https://dx.doi.org/10.4067/S0718-73782018000200133

Davy, Z., Amsler, S., \& Duncombe, K. (2015). Facilitating LGBT Medical, Health and Social Care Content in Higher Education Teaching. Qualitative Research in Education, 4(2), 134-163. http:// dx.doi.org/10.17583/qre.2015.1210

De Boer, A., Pijl, S. J., \& Minnaert, A. (2011). Regular primary schoolteachers' attitudes towards inclusive education: a review of the literature. International Journal of Inclusive Education, 15(3), 331-353. https://doi.org/10.1080/13603110903030089

De Haro Rodríguez, R., Ayala de la Peña, A., \& Del Rey Gil, M. V. (2020). Promoviendo la equidad en los centros educativos: identificar las barreras al aprendizaje y a la participación para promover una educación más inclusiva. Revista Complutense de Educación, 31(3), 341-352. https://doi. org/10.5209/rced.63381

Del Pozo-Armentia, A., Reyero, D., \& Gil Cantero, F. (2020). The pedagogical limitations of inclusive education. Educational Philosophy and Theory, 52(10),1064-1076. https://doi.org/10.1080/0013 1857.2020.1723549

Emmers, E., Baeyens, D., \& Petry, K. (2020). Attitudes and self-efficacy of teachers towards inclusion in higher education. European Journal of Special Needs Education, 35(2), 139-153. https://doi.org/ 10.1080/08856257.2019.1628337

Escalante, E., Repetto, A., \& Mattinello, G. (2012). Exploración y análisis de la actitud hacia la estadística en alumnos de psicología. Liberabit, 18(1), 15-26.

Fernández Batanero, J. M. (2013). Competencias docentes y educación inclusiva. Revista Electrónica de Investigación Educativa, 15(2), 82-99. https://redie.uabc.mx/redie/article/view/445

Garabal-Barbeira, J. (2015). Actitudes de docentes y estudiantes hacia la discapacidad en la Universidade da Coruña. Revista de Estudios e Investigación en Psicología y Educación, 11, 11-19. http://dx.doi. org/10.17979/reipe.2015.0.11.220

Garabal-Barbeira, J., Pousada García, T., Espinosa Breen, P. C., \& Saleta Canosa, J. L. (2018). Las actitudes como factor clave en la inclusión universitaria. Revista Española de Discapacidad, 6(1), 181-198. https://doi.org/10.5569/2340-5104.06.01.09

Garzón, P., Calvo, M. I., \& Orgaz, M. B. (2016). Inclusión educativa. Actitudes y estrategias del profesorado. Inclusión Educativa. Actitudes y estrategias del profesorado. Revista Española de Discapacidad, 4(2), 25-45. https://doi.org/10.5569/2340-5104.04.02.02 
González Cortés, E., \& Roses Campos, S. (2016). ¿Barreras invisibles? Actitudes de los estudiantes universitarios ante sus compañeros con discapacidad. Revista Complutense de Educación, 27(1), 219-235. https://doi.org/10.5209/rev_RCED.2016.v27.n1.45702

González-Castellano, N., Cordón-Pozo, E., Pueyo-Villa, S., \& Colmenero-Ruiz, M. J. (2021). Higher Education teachers' training in attention to SEN students: testing a mediation model. Sustainability, 13(9), 4908. https://doi.org/10.3390/su13094908

González-Gil, F., Martín-Pastor, E., Poy, R., \& Jenaro, C. (2016). Percepciones del profesorado sobre la inclusión: estudio preliminar. Revista Electrónica Interuniversitaria de Formación del Profesorado, 19(3), 11-24. https://doi.org/10.6018/reifop.19.3.219321

González-Rojas, Y., \& Triana-Fierro, D. (2018). Actitudes de los docentes frente a la inclusión de estudiantes con necesidades educativas especiales. Educación y Educadores, 21(2), 200-218. https:// dx.doi.org/10.5294/edu.2018.21.2.2

Granada, M., Pomés, M., \& Sanhueza, S. (2013). Actitud de los profesores hacia la inclusión educativa. Papeles de trabajo-Centro de Estudios Interdisciplinarios en Etnolingüistica y Antropología SocioCultural, 25, 51-59.

Hernández Garre, C., Fernández Martínez, M. del M., Carrión Martínez, J., \& Avilés Soler, B. (2019). La inclusión socioeducativa en la Universidad de Minho. Percepciones y actitudes sobre el alumnado con discapacidad. Revista Complutense de Educación, 30(4), 1097-1112. https://doi.org/10.5209/ rced.60106

Herrera-Seda, C. M, Pérez-Salas, C. P., \& Echeita, G. (2016). Teorías Implícitas y Prácticas de Enseñanza que Promueven la Inclusión Educativa en la Universidad. Instrumentos y Antecedentes para la Reflexión y Discusión. Formación Universitaria, 9(5), 49-64. https://dx.doi.org/10.4067/S071850062016000500006

Ibañez Martín, M. M., Arnaudo, M. F., \& Morresi, S. S. (2018). Inclusión y desempeño: el efecto de políticas educativas en Educación Superior. Un estudio aplicado a la Universidad Nacional del Sur (Argentina). Revista Complutense de Educación, 29(3), 699-717. https://doi.org/10.5209/ RCED.53628

Infante, M. (2010). Desafíos a la Formación Docente: Inclusión Educativa. Estudios Pedagógicos, 36(1), 287-297. http://dx.doi.org/10.4067/S0718-07052010000100016

Ke, Z., Borakova, N. U., \& Valiullina, G. V. (2017). Peculiarities of Psychological Competence Formation of University Teachers in Inclusive Educational Environment. EURASIA Journal of Mathematics Science and Technology Education, 13(8), 4701-4713. https://doi.org/10.12973/ eurasia.2017.00947a

Laca, F. (2005). Actitudes y comportamientos en las situaciones de conflicto. Enseñanza e Investigación en Psicología, 10(1), 117-126.

Lata, D. S., \& Castro, R. M. (2016). El Aprendizaje Cooperativo, un camino hacia la inclusión educativa. Revista Complutense de Educación, 27(3), 1085-1101. https://doi.org/10.5209/rev_RCED.2016. v27.n3.47441

Llorent, V. J., Zych, I., \& Varo-Millán, J. C. (2020). University academic personnel's vision of inclusive education in Spanish universities. Culture and Education, 32(1), 147-181. https://doi.org/10.108 $0 / 11356405.2019 .1705593$ 
Manterola, C., Astudillo, P., Arias, E., \& Claros, N. (2013). Revisiones sistemáticas de la literatura. Qué se debe saber acerca de ellas. Cirugía Española, 91(3), 149-155.

Moriña, A. (2017). Inclusive education in higher education: challenges and opportunities. European Journal of Special Needs Education, 32(1), 3-17. https://doi.org/10.1080/08856257.2016.1254964

Moriña, A. (2020). Faculty members who engage in inclusive pedagogy: methodological and affective strategies for teaching. Teaching in Higher Education, 1-16. https://doi.org/10.1080/13562517.2 020.1724938

Navarro-Aburto, B., Arriagada Puschel, I., Osse-Bustingorry, S., \& Burgos-Videla, C. (2016). Adaptaciones curriculares: similitudes y diferencias de su implementación en la facultad chilena. Revista Electrónica Educare, 20(1), 1-18. https://doi.org/10.15359/ree.20-1.15

Ocampo, A. (2012). Inclusión de estudiantes en situación de discapacidad a la educación superior. Desafíos y oportunidades. Revista Latinoamericana de Educación Inclusiva, 6(2), 227-239.

Ortiz Colón, A. M., Agreda Montoro, M., \& Colmenero-Ruiz, M. J. (2018). Towards inclusive higher education in a global context. Sustainability, 10(8), 2670. https://doi.org/10.3390/su10082670

Pacheco Ruíz, F. (2002). Actitudes. Eúphoros, 5, 173-186.

Paz-Maldonado, E. (2018a). Competencias del profesorado universitario para la atención a la diversidad en la educación superior. Revista Latinoamericana de Educación Inclusiva, 12(2), 115-131. http:// dx.doi.org/10.4067/S0718-73782018000200115

Paz-Maldonado, E. (2018b). La formación del profesorado universitario para la atención a la diversidad en la educación superior. IE Revista De Investigación Educativa de la REDIECH, 9(16), 67-82. https://doi.org/10.33010/ie_rie_rediech.v9i16.108

Paz-Maldonado, E. (2020a). Una aproximación teórica sobre la educación inclusiva en Honduras: avances, obstáculos y desafíos. Revista Brasileira de Educação Especial, 26(3), 371-386. https://doi. org/10.1590/1980-54702020v26e0049

Paz-Maldonado, E. (2020b). Inclusión educativa del alumnado en situación de discapacidad en la educación superior: una revisión sistemática. Teoría de la Educación, 32(1), 123-146. http://dx.doi. org/10.14201/teri.20266

Pegalajar Palomino, M., \& Colmenero-Ruiz, M. (2014). Actitudes del docente de centros de educación especial hacia la inclusión educativa. Enseñanza \& Teaching, 32(2), 195-213. http://dx.doi. org/10.14201/et2014321195213

Pereira, R. R., Faciola, R. A., Pontes, F. A. R., Ramos, M. F. H., \& Silva, S. S. C. (2020). Students with disabilities at the Federal University of Pará: difficulties and suggestions for improvement. Revista Brasileira de Educação Especial, 26(3), 253-268. https://doi.org/10.1590/1980-54702020v26e0087

Poker, R. B., Valentim, F. O. D., \& Garla, I. A. (2018). Inclusion in higher education: the perception of teachers of a public institution in the interior of the state of São Paulo. Psicologia Escolar e Educacional, 22(Spe), 127-134. https://doi.org/10.1590/2175-3539/2018/010

Polo Sánchez, M. T., Fernández-Jiménez, C., \& Fernández Cabezas, M. (2017). The Attitudes of Different Partners Involved in Higher Education towards Students with Disabilities. International Journal of Disability, Development and Education, 65(4), 442-458. https://doi.org/10.1080/10349 12X.2017.1406066 
Rodríguez-Martín, A., \& Álvarez-Arregui, E. (2015). Universidad y discapacidad. Actitudes del profesorado y de estudiantes. Perfiles Educativos, 37(147), 86-102. https://doi.org/10.1016/j. pe.2014.09.001

Rodríguez-Martín, A., Álvarez-Arregui, E., \& García-Ruiz, R. (2014). La atención a la diversidad en la universidad: El valor de las actitudes. REOP. Revista Española de Orientación y Psicopedagogía, 25(1), 44-61. https://doi.org/10.5944/reop.vol.25.num.1.2014.12012

Saloviita, T. (2020). Attitudes of Teachers Towards Inclusive Education in Finland. Scandinavian Journal of Educational Research, 64(2), 270-282. https://doi.org/10.1080/00313831.2018.1541819

Sánchez Díaz, M. N., \& Morgado Camacho, B. (2021). Docentes universitarios inclusivos: qué les caracteriza y cómo conciben la discapacidad. Siglo Cero, 52(1), 27-43. https://doi.org/10.14201/ scero20215212743

Sandoval Mena, M., Simón Rueda, C., \& Márquez Vásquez, C. (2019). ¿Aulas inclusivas o excluyentes?: barreras para el aprendizaje y la participación en contextos universitarios. Revista Complutense de Educación, 30(1), 261-276. https://doi.org/10.5209/RCED.57266

Sanhueza, S., Granada, M., \& Bravo, L. (2012). Actitudes del profesorado de Chile y Costa Rica hacia la inclusión educativa. Cadernos de Pesquisa, 42(147), 884-899. https://doi.org/10.1590/S010015742012000300013

Seale, J. (2017). From the voice of a 'socratic gadfly': a call for more academic activism in the researching of disability in postsecondary education. European Journal of Special Needs Education, 32(1), 153169. https://doi.org/10.1080/08856257.2016.1254967

Sevilla Santo, D. E., Martín Pavón, M. J., \& Jenaro, C. (2017). Percepciones sobre la educación inclusiva: la visión de quienes se forman para docentes. CPU-e. Revista de Investigación Educativa, 25, 83-113.

Tejeda Cerda, P. (2019). La evaluación educativa en estudiantes en situación de discapacidad en la universidad: desafíos y propuestas. Estudios Pedagógicos, 45(2), 169-178. https://doi.org/10.4067/ S0718-07052019000200169

Urrútia, G., \& Bonfill, X. (2010). Declaración PRISMA: una propuesta para mejorar la publicación de revisiones sistemáticas y metaanálisis. Medicina Clínica, 135(11), 507-511. https://doi. org/10.1016/j.medcli.2010.01.015

Yada, A., \& Savolainen, H. (2017). Japanese in-service teachers' attitudes toward inclusive education and self-efficacy for inclusive practices. Teaching and Teacher Education, 64, 222-229. https://doi. org/10.1016/j.tate.2017.02.005

Recebido em: 16/01/2021

Reformulado em: 10/06/2021

Aprovado em: 20/07/2021 